\title{
Effects of Repeated Cocaine Exposure on Habit Learning and Reversal by $\mathrm{N}$-Acetylcysteine
}

\author{
Laura H Corbit*,', Billy C Chieng ${ }^{2}$ and Bernard W Balleine ${ }^{2}$ \\ 'School of Psychology, University of Sydney, Sydney, NSW, Australia; 'Brain and Mind Research Institute, University of Sydney, Sydney, NSW, \\ Australia
}

\begin{abstract}
Exposure to drugs of abuse can result in a loss of control over both drug- and nondrug-related actions by accelerating the transition from goal-directed to habitual control, an effect argued to reflect changes in glutamate homeostasis. Here we examined whether exposure to cocaine accelerates habit learning and used in vitro electrophysiology to investigate its effects on measures of synaptic plasticity in the dorsomedial (DMS) and dorsolateral (DLS) striatum, areas critical for actions and habits, respectively. We then administered $\mathrm{N}$-acetylcysteine (NAC) in an attempt to normalize glutamate homeostasis and hence reverse the cellular and behavioral effects of cocaine exposure. Rats received daily injections of cocaine $(30 \mathrm{mg} / \mathrm{kg})$ for 6 days and were then trained to lever press for a food reward. We used outcome devaluation and whole-cell patch-clamp electrophysiology to assess the behavioral and cellular effects of cocaine exposure. We then examined the ability of NAC to reverse the effects of cocaine exposure on these measures. Cocaine treatment produced a deficit in goal-directed action, as assessed by outcome devaluation, and increased the frequency of spontaneous and miniature excitatory postsynaptic currents (EPSCS) in the DMS but not in the DLS. Importantly, NAC treatment both normalized EPSC frequency and promoted goal-directed control in cocaine-treated rats. The promotion of goal-directed control has the potential to improve treatment outcomes in human cocaine addicts.

Neuropsychopharmacology (2014) 39, I893-190I; doi:I0.1038/npp.2014.37; published online I2 March 2014
\end{abstract}

\section{INTRODUCTION}

Repeated exposure to drugs of abuse causes a wide range of long-lasting neural adaptations (Kalivas, 2009; Kauer and Malenka, 2007). Nevertheless, although an increasing number of studies have modeled factors that promote sustained drug seeking and taking, there has been little systematic research into how drug exposure influences decision making more generally. Extensive research using sensitization paradigms has been conducted to model the early neural changes that accompany drug exposure; however, to date, this work has not established whether these changes are sufficient to lead to general executive dysfunction. As an important advance in this regard, it has recently been shown that psychostimulant exposure accelerates the loss of volitional control over reward seeking, causing abnormally rapid development of response habits that are insensitive to changes in the value of the outcomes they produce (LeBlanc et al, 2013; Nelson and Killcross, 2006; Nelson and Killcross, 2013; Nordquist et al, 2007; Schoenbaum and Setlow, 2005; Zapata et al, 2010). It remains unknown, however, how

\footnotetext{
*Correspondence: Dr LH Corbit, School of Psychology, Brennan MacCallum Building (AI8), University of Sydney, Sydney, NSW 2006, Australia, Tel: +6129451 7074, Fax: +61 29036 5223,

E-mail: laura.corbit@sydney.edu.au

Received 18 December 2013; revised 5 February 2014; accepted 7 February 2014; accepted article preview online 17 February 2014
}

psychostimulants alter neural activity in structures that mediate goal-directed and habit learning to produce this change in behavioral control.

Understanding the neural changes that underlie the effects of drugs on decision making is critical for establishing the necessary conditions underlying these behavioral changes and for ameliorating or, ideally, reversing these changes. Reports that the induction of behavioral sensitization can be prevented by attenuating synaptic glutamate signaling during drug exposure are of considerable interest in this regard (Li et al, 1997; Madayag et al, 2007). Chronic drug exposure not only alters synaptic glutamate receptors but also impairs glutamate homeostasis by altering the balance between synaptic and extrasynaptic glutamate release and elimination (Kalivas, 2009). Basal extracellular glutamate levels are reduced following drug exposure and withdrawal and this reduces the inhibitory tone via metabotropic receptors and increases synaptic release (Moran et al, 2005). This is due, in part, to downregulation of cystine-glutamate exchange and glutamate transporters that normally regulate extracellular glutamate levels. Drugs that restore this balance have been shown to reduce drugseeking behaviors. For example, treatment with $\mathrm{N}$-acetylcysteine (NAC), which activates cystine-glutamate exchange, prevents behavioral sensitization and reinstatement of cocaine self-administration (Baker et al, 2003; Knackstedt et al, 2010; Madayag et al, 2007; Zhou and Kalivas, 2008). Thus, we hypothesized that NAC may also prevent the 
abnormally rapid formation of habits following stimulant exposure and help to restore normal decision-making processes.

The following experiments examined whether pretraining exposure to cocaine leads to earlier habitual control of performance or a more general learning deficit. The in vitro electrophysiological experiments examined cocaine-induced changes in excitatory postsynaptic currents (EPSCs) in the dorsomedial (DMS) and dorsolateral (DLS) regions of the dorsal striatum previously shown to underlie goal-directed and habit learning, respectively (see Balleine and O'Doherty, 2010 for review). Finally, we investigated the ability of NAC to reverse cocaine-induced alterations in synaptic plasticity in animals with a history of cocaine exposure and then examined whether cotreatment with NAC could prevent the effects of cocaine on subsequent learning.

\section{MATERIALS AND METHODS}

Experiment 1: Does Repeated Cocaine Exposure Accelerate the Transition from Goal-Directed to Habitual Action Control?

Subjects and apparatus. A total of 17 experimentally naive male Long-Evans rats (Monash, Clayton, VIC, Australia) served as subjects. Rats weighed between 225 and $250 \mathrm{~g}$ at the beginning of the experiment, were housed in groups of $2-3$, and handled daily for 1 week before drug treatments. Training and testing took place in Med Associates (St Albans, VT) operant chambers housed within sound- and light-attenuating shells. The chambers were equipped with a pellet dispenser that delivered one $45 \mathrm{mg}$ pellet when activated (grain-based formula; Bio-Serv, Frenchtown, NJ) and a syringe pump that delivered $0.1 \mathrm{ml}$ of a $20 \%$ sucrose solution when activated. The chambers contained two retractable levers that could be inserted to the left and the right of the magazine. A $3 \mathrm{~W}, 24 \mathrm{~V}$ houselight mounted on the wall across from the levers and magazine illuminated the chamber. Microcomputers equipped with MED-PC software (Med Associates) controlled the equipment and recorded responses.

Cocaine treatment. Because extended training with natural rewards can produce habitual performance (Adams, 1982), within drug self-administration paradigms it is difficult to isolate the effects of repeated performance of the target response from the effects of the drug, and hence in the current study we examined the effects of experimenter-administered drug on subsequent learning. Rats were divided into groups and received either saline or cocaine i.p., daily for 6 days. Rats in the cocaine group received daily injections of $30 \mathrm{mg} / \mathrm{kg}$ of cocaine hydrochloride (National Measurement Institutes, Lindfield, NSW, Australia). Similar treatment has been shown elsewhere to induce locomotor sensitization and produce changes in glutamate signaling (Ghasemzadeh et al, 2003; Madayag et al, 2007). The volume of the injection was always $1 \mathrm{ml} / \mathrm{kg}$ and controls received the same volume of saline. Rats were returned to the home cage following each injection. Rats were weighed daily and gradually food restricted during the 7-day delay between the final injection and the beginning of behavioral training.
Behavioral methods. Although an individual rat was only trained to respond for one outcome (pellets or sucrose, counterbalanced), all rats were exposed to both outcomes in the home cage before training to reduce novelty and hence ensure consumption during devaluation testing where both foods were used to generate the devalued and nondevalued conditions.

Magazine training. Rats received 1 day of magazine training in which the training outcome (either pellets or sucrose, counterbalanced) was delivered on a RT-60 s schedule for 30 min.

Instrumental training. Rats were trained to make a single lever-press response. Each response was reinforced in the initial two sessions. Thereafter, responding was reinforced according to variable interval (VI) schedules with 2 days on VI15, 2 days on VI30, and 4 days on VI60 before the first test. All sessions terminated after 30 outcomes were delivered and thus a maximum of 30 outcomes were earned in each session and a total of 300 outcomes before the first tests. Rats then received 8 further training sessions where responding was rewarded according to the VI60 schedule, earning a total of 540 outcomes before the late tests.

Devaluation testing. Rats had free access to either pellets or sucrose for $1 \mathrm{~h}$ in separate feeding cages (Plexiglas tubs measuring $33 \times 21 \times 19 \mathrm{~cm}$ ) before a 5-min extinction test in which the lever was present but no outcomes were delivered. In the first test, half of the animals were prefed their earned outcome (eg, pellets; devalued), whereas the remaining animals were prefed the alternative outcome (eg, sucrose; nondevalued). The rats were given one retraining session on the VI60 schedule before a second devaluation test in which they were prefed the opposite food, thus reversing, and counterbalancing the order of, the devaluation condition. The late tests used the same procedures.

\section{Experiment 2: Does Repeated Cocaine Exposure Alter Glutamate Release in Dorsolateral and Dorsomedial Striatum?}

Subjects and apparatus. A total of 32 experimentally naive male Long-Evans rats (Monash) served as subjects housed as in experiment 1 . Rats were between 6 and 7 weeks of age ( $\sim 200 \mathrm{~g})$ at the time of drug treatment and between 8 and 9 weeks of age at the time of the electrophysiology experiments.

Cocaine and NAC treatments. Rats received either cocaine $(30 \mathrm{mg} / \mathrm{kg})$ or saline for 6 days, followed by 7 days of rest, as in experiment 1 . To examine whether cotreatment with NAC (Sigma, Castle Hill, NSW, Australia) would normalize the changes to glutamate release observed in the DMS, additional rats were divided into four groups assigned to receive either cocaine or saline and either 0 or $120 \mathrm{mg} / \mathrm{kg}$ of NAC daily for 6 days, followed by 7 days of rest, as in the experiments above.

Brain slice preparation. Rats were killed under deep anesthesia by isoflurane inhalation ( $4 \%$ in air), decapitated, 
and the brain removed. Coronal brain slices ( $300 \mu \mathrm{m}$ thick) containing the posterior dorsomedial and anterior dorsolateral striatum were cut using a vibrating tissue slicer (Leica VT1000S, Germany) in ice-cold oxygenated sucrose cutting solution containing (in $\mathrm{mM}$ ): 241 sucrose, 28 $\mathrm{NaHCO}_{3}, 11$ glucose, $1.4 \mathrm{NaH}_{2} \mathrm{PO}_{4}, 3.3 \mathrm{KCl}, 0.2 \mathrm{CaCl}_{2}$, and $7 \mathrm{MgCl}_{2}$. Slices were hemisected at midline and maintained at $33{ }^{\circ} \mathrm{C}$ in a submerged chamber containing physiological saline with composition (in $\mathrm{mM}$ ): $126 \mathrm{NaCl}, 2.5 \mathrm{KCl}, 1.4$ $\mathrm{NaH}_{2} \mathrm{PO}_{4}, 1.2 \mathrm{MgCl}_{2}, 2.4 \mathrm{CaCl}_{2}, 11$ glucose, and $25 \mathrm{NaHCO}_{3}$, and equilibrated with $95 \% \mathrm{O}_{2}$ and $5 \% \mathrm{CO}_{2}$.

Electrophysiological recording. After equilibrating slices for $1 \mathrm{~h}$, a slice was then transferred to a recording chamber and visualized under a microscope (Olympus BX50WI) using differential interference contrast Dodt tube optics, and superfused continuously $(1.5 \mathrm{ml} / \mathrm{min})$ with oxygenated physiological saline at $33^{\circ} \mathrm{C}$. Whole-cell patch-clamp recordings were made in medium-sized spiny neurons (MSNs) using electrodes (2-5 M $\Omega$ ) containing internal solution (in $\mathrm{mM}$ ): 127 cesium methanesulfonate, $20 \mathrm{NaCl}, 20$ HEPES, 0.4 EGTA, 5 tetraethylammonium chloride, 3 QX314 chloride, 2.5 Mg-ATP, and 0.33 Na-GTP, pH 7.3, osmolarity 285-290 mOsm/l. Biocytin (0.1\%; Sigma-Aldrich, St Louis, MO) was routinely added to the internal solution for marking the sampled neurons during recording. Data acquisition was performed with an Axopatch $200 \mathrm{~A}$ amplifier (Molecular Devices, Sunnyvale, CA), connected to a Macintosh computer and interface ITC-16 (Instrutech, Long Island, NY). Whole-cell currents were sampled at $5 \mathrm{kHz}$ (low-pass filter $2 \mathrm{kHz}$, Axograph X; Molecular Devices) and voltage-clamped at $-60 \mathrm{mV}$, without liquid junction potential correction. Series resistance $(<25 \mathrm{M} \Omega)$ was compensated by $80 \%$ and monitored. Stock solutions of all drugs were diluted to working concentrations in the extracellular solution immediately before use and applied by continuous superfusion. Spontaneous EPSCs (sEPSCs) were sampled in the presence of picrotoxin $(100 \mu \mathrm{M}$; Sigma-Aldrich), DPCPX (200 nM; Tocris, Bristol, UK), and CGP55845 (1 $\mathrm{MM}$; Tocris), whereas miniature EPSCs (mEPSCs) were sampled with additional tetrodotoxin $(1 \mu \mathrm{M}$; Ascent Scientific, Bristol, UK) present. Data (5 min length of recording) were analyzed in Axograph $\mathrm{X}$ (Molecular Devices) and statistical significance evaluated using ANOVA and Bonferroni post hoc tests.

\section{Experiment 3: Does Cotreatment with NAC Prevent Cocaine Exposure from Accelerating Habit Learning?}

Subjects and apparatus. A total of 64 experimentally naive male Long-Evans rats (Monash) served as subjects housed as in experiment 1 .

Cocaine sensitization and NAC treatments. To examine whether NAC could prevent the accelerated habit learning observed following cocaine exposure, rats were assigned to receive either cocaine or saline and either 0,60 , or $120 \mathrm{mg} /$ $\mathrm{kg}$ of NAC administered i.p. $2 \mathrm{~h}$ before cocaine treatment (doses and timing based on previous studies; Madayag et al, 2007; Moussawi et al, 2011). Groups receiving each dose of NAC were further divided and received either saline or $30 \mathrm{mg} / \mathrm{kg}$ cocaine $2 \mathrm{~h}$ after the NAC treatment, thus generating six groups. Rats were treated for 6 consecutive days and were handled and gradually food restricted for a further 7 days before beginning instrumental training.

Behavioral methods. Instrumental training was conducted as described in experiment 1 above except that a single set of devaluation tests was conducted after rats had earned 540 outcomes. Based on the results of experiment 1 , cocaine-treated but not saline-treated rats were expected to show habitual performance, allowing us to assess any effects of cotreatment with NAC.

\section{RESULTS}

\section{Experiment 1: Cocaine Exposure Accelerates Habitual Control}

Instrumental training. Figure 1a shows that both groups increased lever pressing across training sessions. A mixed ANOVA showed a significant effect of day $(\mathrm{F}(17,238)=$ $10.6, p<0.001)$, but no effect of group $(\mathrm{F}(1,14)=1.7$, $p>0.05)$, and no interaction between these factors
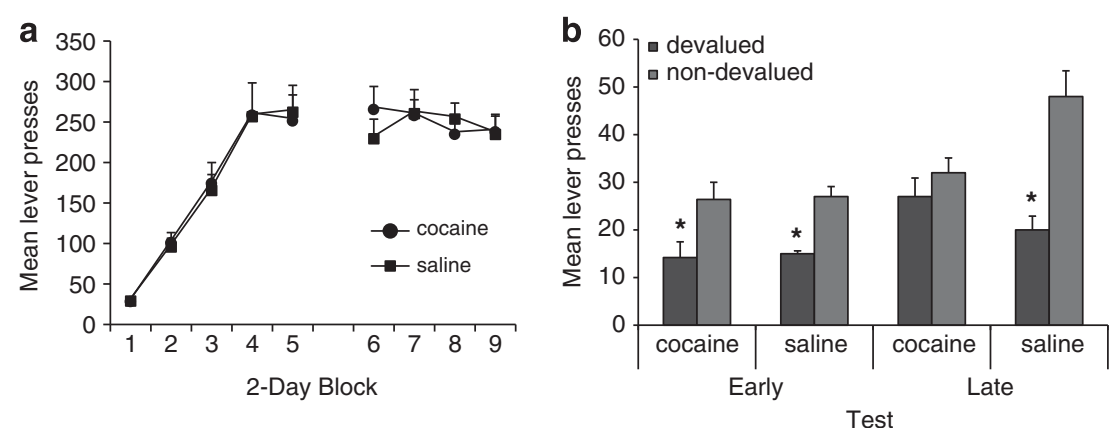

Figure I Effects of cocaine on instrumental conditioning and sensitivity to outcome devaluation. (a) Mean ( + SEM) lever presses across 2-day blocks of instrumental training. Lever-press rates increased across training but there were no group differences in lever-press rates indicating equivalent instrumental performance during training. (b) Mean (+SEM) lever presses following prefeeding of the earned (devalued) or control (nondevalued) food outcome in tests conducted relatively early (when rats had earned 300 rewards) or late (when rats had earned 540 rewards) in training. In the Early test, both groups showed equivalent sensitivity to outcome devaluation decreasing performance following prefeeding of the earned outcome. In the Late test, although rats previously treated with saline remained sensitive to devaluation, performance of those previously treated with cocaine was no longer sensitive to devaluation, demonstrating that performance in these animals had come under habitual control. $\mathrm{N}=8-9$ rats per group, *significant devaluation effect. 
$(\mathrm{F}(17,238)=0.4, p>0.05)$. Thus, both groups showed equivalent instrumental performance in training.

Devaluation testing. The results of the devaluation tests are presented in Figure 1b. ANOVA revealed a main effect of devaluation such that responding was significantly lower in the devalued condition $(\mathrm{F}(1,14)=45.5, p<0.01)$ and an effect of test (early $v s$ late; $F(1,14)=16.3, p<0.01$ ). There was no main effect of group $(\mathrm{F}(1,14)=1.1, p>0.05)$; however, there was an interaction between devaluation and group $(\mathrm{F}(1,14)=7.4, p<0.05)$ and although there was no interaction between test and devaluation $(\mathrm{F}(1,14)=1.7$, $p>0.05)$, there was a three-way interaction indicating that sensitivity to devaluation differed between groups according to test $(F(1,14)=9.5, p<0.01)$. Further analyses revealed that in the early test, there was an effect of devaluation $(\mathrm{F}(1,14)=28.3, p<0.01)$, no effect of group $(\mathrm{F}(1,14)=0.01, p>0.05)$, and no interaction $(\mathrm{F}(1,14)=0.4$, $p>0.05$ ), indicating that both groups were similarly sensitive to devaluation. In contrast, in the late test, there was again an effect of devaluation $(\mathrm{F}(1,14)=21.7, p<0.01)$, and although there was no effect of group $(F(1,14)=1.2$, $p>0.05)$ there was an interaction between these factors $(\mathrm{F}(1,14)=10.5, p<0.01)$. The post hoc tests (Bonferroni) confirmed a significant devaluation effect in saline- but not cocaine-treated rats.

\section{Experiment 2: Cocaine Exposure Alters Glutamate Release in the DMS}

Figure 2a displays representative raw traces from cells in the DMS of saline- and cocaine-treated rats. These data are summarized in Figure $2 \mathrm{~b}-\mathrm{e}$. As shown in Figure 2, compared with the saline group, cocaine treatment significantly increased the frequency of sEPSC in the pDMS but not the DLS. In the DMS, there was a significant effect of cocaine on sEPSC frequency (Figure $2 \mathrm{~b} ; \mathrm{F}(1,14)=11.3$, $p<0.01$ ) but not amplitude (Figure 2c; $F(1,14)=0.1$, $p>0.05)$. In the DLS, there was no effect of cocaine on either sEPSC frequency $(\mathrm{F}(1,10)=0.3, p>0.05)$ or amplitude $(\mathrm{F}(1,10)=0.2, p>0.05)$. In the presence of tetrodotoxin used to examine terminal release of glutamate, mEPSC recordings confirmed presynaptic changes in excitatory terminals in the DMS induced by cocaine treatment. The pattern of results was similar to that seen for sEPSCs. In the DMS, there was a significant effect of cocaine on mEPSC frequency (Figure 2d; $\mathrm{F}(1,11)=7.3, p<0.05$ ) but not amplitude (Figure 2e; $F(1,11)=0.2, p>0.05$ ). In the DLS, there was no effect of cocaine on either MEPSC frequency $(\mathrm{F}(1,8)=0.04, \quad p>0.05) \quad$ or amplitude $\quad(\mathrm{F}(1,8)=1.5$, $p>0.05)$.

\section{NAC Prevents Cocaine-Induced Effects on Glutamate Release in the DMS}

In a separate group of rats, the cocaine-induced elevation of sEPSCs and mEPSCs in the DMS was further examined with and without prior NAC treatment. Figure 3a shows representative traces from the different groups. These data revealed that both sEPSC (Figure $3 \mathrm{~b}$ ) and mEPSC (Figure 3d) frequency but not amplitude (Figure $3 \mathrm{c}$ and e) were increased by cocaine treatment, and NAC treatment reversed this effect. ANOVA for sEPSCs confirmed a significant effect of cocaine in saline-treated $(F(1,20)=11.8$, $p<0.01)$ but not NAC-treated animals $(\mathrm{F}(1,16)=1.4$, $p>0.05)$. The amplitude of sEPSCs was unaffected by cocaine treatment in either saline-treated $(\mathrm{F}(1,20)=0.3$, $p>0.05)$ or NAC-treated animals $(\mathrm{F}(1,16)=0.3, p>0.05)$. Again, a similar pattern was observed for mEPSCs confirming a presynaptic effect of cocaine. ANOVA demonstrated a significant effect of cocaine in saline-treated $(\mathrm{F}(1,17)=10.2$, $p<0.01)$ but not NAC-treated animals $(\mathrm{F}(1,12)=2.0$, $p>0.05)$. The amplitude of sEPSCs was unaffected by cocaine treatment in either saline-treated $(\mathrm{F}(1,17)=0.02$, $p>0.05)$ or NAC-treated animals $(\mathrm{F}(1,12)=0.15, p>0.05)$.

\section{Experiment 3: NAC Prevents the Acceleration of Habit Learning Induced by Cocaine}

Instrumental training. Figure $4 \mathrm{a}$ shows that all groups increased lever pressing across training sessions. A mixed ANOVA showed a significant effect of day $(F(17,986)=$ $131.7, p<0.01)$, but no effect of group $(F(5,58)=1.3$, $p>0.05)$, and no interaction between these factors $(\mathrm{F}(85,986)=1.4, p>0.05)$. Thus, all groups showed equivalent instrumental performance during training.

Devaluation testing. The results of the devaluation tests are presented in Figure 4. ANOVA revealed no overall effect of cocaine $(\mathrm{F}(1,58)=0.03, p>0.05)$ or $\mathrm{NAC}(\mathrm{F}(2,58)=0.13$, $p>0.05)$ but a main effect of devaluation $(\mathrm{F}(1,58)=71.4$, $p<0.01)$ and interactions between both cocaine and NAC and devaluation $(\mathrm{F}(1,58)=5.8, \quad p<0.05 ; \mathrm{F}(2,58)=4.8$, $p<0.05$, respectively). There was also a three-way interaction between these factors $(F(2,58)=3.2, p<0.05)$. Simple effects analyses found that for animals treated with saline, there was a significant effect of devaluation $(\mathrm{F}(1,28)=36.2$, $p<0.01)$, no effect of NAC $(\mathrm{F}(2,28)=1.8, p>0.05)$, and no interaction between these factors $(\mathrm{F}(2,28)=0.07, p>0.05)$, indicating there was a similar devaluation effect in all groups (Figure $4 \mathrm{~b}$ ). In contrast, for animals treated with cocaine (Figure 4c), there was no main effect of NAC $(\mathrm{F}(2,30)=1.1, \quad p>0.05)$, but an effect of devaluation $(\mathrm{F}(1,30)=41.3, p<0.01)$, and a significant interaction between these factors $(\mathrm{F}(2,30)=17.4, p<0.01)$, indicating that the effect of cocaine on sensitivity to devaluation was altered by NAC treatment. The post hoc tests found no devaluation effect in rats treated with cocaine and saline but a significant devaluation effect in rats treated with cocaine and either 60 or $120 \mathrm{mg} / \mathrm{kg}$ of NAC.

\section{DISCUSSION}

The current experiments found that, in instrumental conditioning, cocaine exposure accelerated habit learning in a similar manner to amphetamine (Nelson and Killcross, 2006; Nordquist et al, 2007). Whereas cocaine-treated rats showed similar sensitivity to outcome devaluation as drugnaive rats early in training, they differed after further training, showing insensitivity to devaluation and therefore more rapid habit acquisition than controls. Exposure to psychostimulants leads to lasting neural changes in cortical, limbic, and striatal regions areas implicated in instrumental 

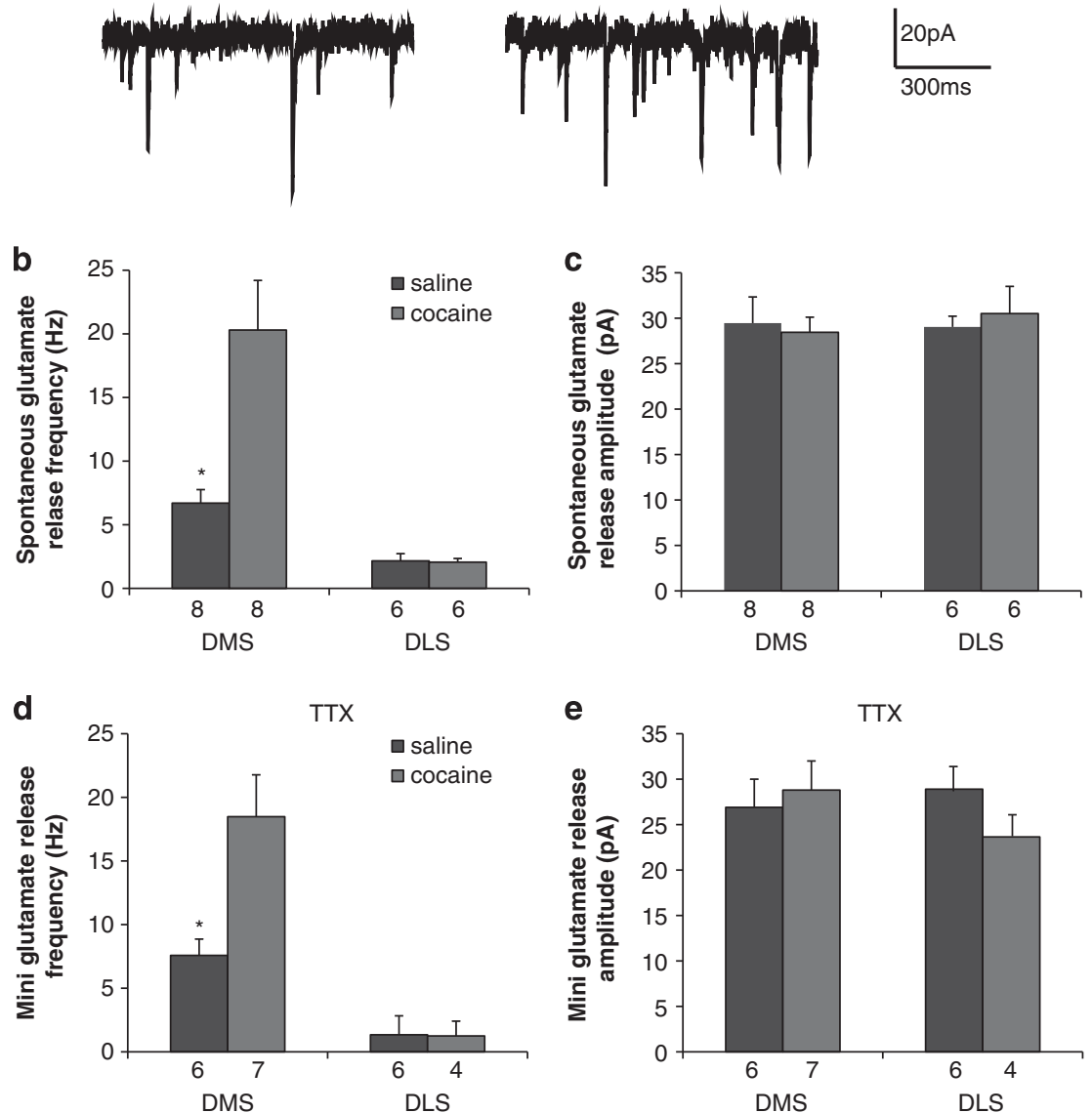

Figure 2 Cocaine increased glutamate release in pDMS but not DLS. (a) Representative raw traces of sEPSCs from saline- and cocaine-treated animals. Mean ( + SEM) whole-cell recordings of sEPSC frequency (b) and amplitude (c) in pDMS and DLS from saline- and cocaine-treated rats. In the presence of TTX $(I \mu M)$, the cocaine-mediated increase in mean mEPSC frequency persisted in the pDMS (d) whereas its corresponding amplitude remained unchanged (e). No effects of cocaine were seen in the DLS. The numbers of cells recorded are indicated below the corresponding mean for that condition; $N=4-8$ neurons. *Significant difference, $p<0.05$.

learning and performance (Paulson and Robinson, 1995; Pierce and Kalivas, 1997; Vanderschuren and Kalivas, 2000), and in experiment 2 we looked for direct evidence of such changes in striatal regions known to support goal-directed learning and habit learning. Previous work has focused on neural adaptations in the nucleus accumbens in increased susceptibility to relapse (Kalivas, 2009; Kauer and Malenka, 2007; Kupchik et al, 2012), and although the accumbens plays an important role in instrumental performance (Corbit and Balleine, 2011; Corbit et al, 2001; Laurent et al, 2012), evidence suggests that regions of the dorsal striatum are more critical for acquisition of both goaldirected and habitual actions. For example, pretraining, post-training, or reversible inactivation of the DMS disrupts sensitivity to outcome devaluation or manipulations of the response-outcome (R-O) contingency (Corbit and Janak, 2010; Yin et al, 2005a, b), whereas lesions or reversible inactivation of the DLS prevents the acquisition or expression of habits (Corbit et al, 2012; Yin et al, 2004). We hypothesized, therefore, that in addition to effects reported in the nucleus accumbens, cocaine could alter glutamate signaling in dorsal striatal regions that support goal-directed and habit learning and thus alter these forms of learning. Indeed, in experiment 2 we found that cocaine exposure alters presynaptic glutamatergic activity, increasing sEPSCs and mEPSCs specifically in the DMS of rats exposed to cocaine. It appears likely that this change in the normal neural activity in the DMS altered the goaldirected learning process normally supported by the DMS (Corbit and Janak, 2010; Yin et al, 2005b), in a manner resulting in early control by the habit system in cocainetreated rats.

In support of this suggestion, cocaine treatment appeared to induce little if any change in activity within the DLS. This finding is of great interest because changes in the balance between goal-directed and habitual control can be achieved by bolstering habit learning or by impairing goal-directed learning. Altered activity in the DMS rather than DLS points to a failure of goal-directed control at least in the case of cocaine exposure. Although the exact nature of this transition requires further study, impairments in the ability to induce LTP and LTD in the prefrontal cortex to nucleus accumbens core pathway have been reported following cocaine exposure (Moussawi et al, 2009) and similar changes in afferent pathways to the DMS could account for weakened goal-directed learning in the current case. 


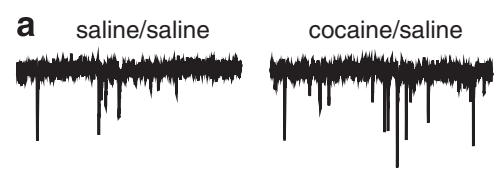

b

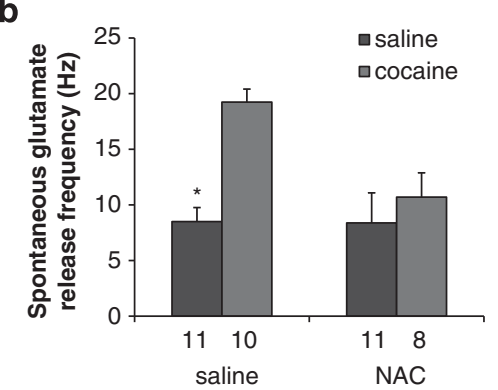

d

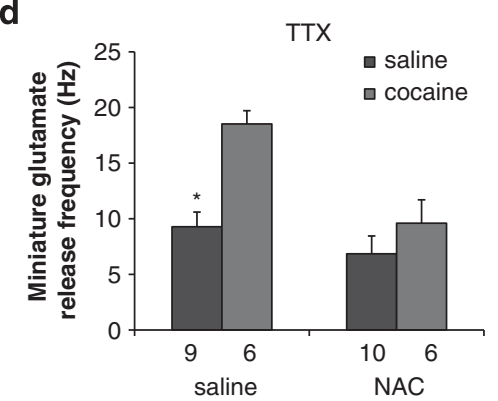

saline/NAC

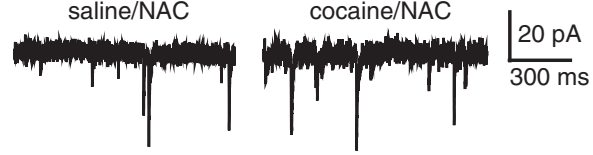

C

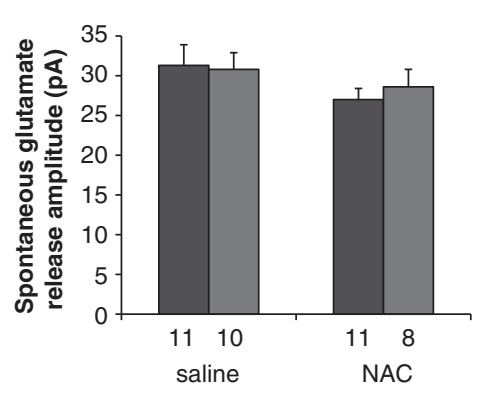

e

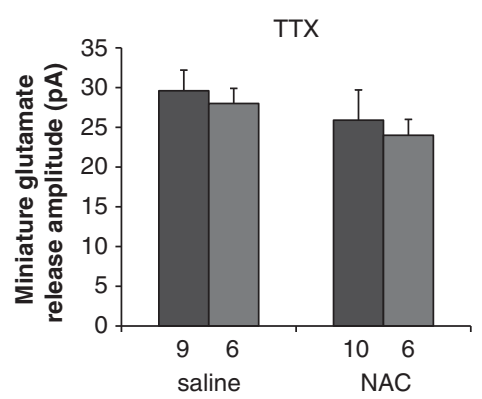

Figure 3 Effects of NAC treatment on cocaine-induced changes in glutamate release in pDMS. (a) Representative raw traces of sEPSCs from animals in the four groups. Mean ( + SEM) whole-cell recordings of sEPSC and mEPSC frequency and amplitude. Cocaine treatment was again found to increase both mean sEPSC and mEPSC frequency (b, $d$ ) and these effects were blocked by NAC treatment. The mean sEPSC (c) and mEPSC (e) amplitude were again unaffected by cocaine. The numbers of cells recorded are indicated below the corresponding mean for that condition; $N=6-11$. *Significant difference, $p<0.05$.

\section{Neural Changes That Accompany Sensitization and the Effects of NAC}

A critical role for glutamate signaling in the induction of sensitization is perhaps best demonstrated by findings that blocking AMPA or NMDA receptors with antagonists (Ghasemzadeh et al, 2003; Li et al, 1997; Stewart and Druhan, 1993) during exposure to the psychostimulant drug prevents sensitization. In addition to the direct effects on synaptic ionotropic glutamate receptors, chronic drug exposure disrupts glutamate homeostasis by altering the balance between synaptic and extrasynaptic glutamate release and elimination (see Kalivas, 2009). Basal extracellular glutamate levels are reduced following drug exposure and withdrawal and this reduces the inhibitory tone via metabotropic receptors (mGluR2/3) and increases synaptic release. This is due, in part, to downregulation of cystine-glutamate exchange and glutamate transporters that normally regulate extracellular glutamate levels. Drugs that restore this balance have been shown to reduce drugseeking behaviors. For example, treatment with NAC that activates cystine-glutamate exchange or with ceftriaxone that restores glutamate transporter levels (eg, GLT-1) prevents behavioral sensitization and reinstatement of cocaine self-administration (Knackstedt et al, 2010; Madayag et al, 2007; Zhou and Kalivas, 2008). Furthermore, treatment with NAC reduces cocaine craving in humans (Amen et al, 2011) and restores the ability to induce both
LTP and LTD that may be critical for normal learning to occur (Moussawi et al, 2009).

We therefore hypothesized that the changes in glutamatergic activity, particularly the changes in sEPSCs and mEPSCs in the DMS of rats exposed to cocaine, could be reversed by NAC. Furthermore, drugs that restore glutamate homeostasis, such as NAC, have been shown to block not only synaptic changes but also cue and cocaine-primed reinstatement of cocaine seeking (Kupchik et al, 2012), suggesting that NAC might both normalize glutamate activity within the DMS and restore goal-directed control. The results of experiments 2 and 3 confirmed this hypothesis, demonstrating that treatment with NAC both normalized EPSCs in the DMS and promoted goal-directed control in cocaine-treated rats; indeed, rats that received NAC before cocaine treatment were indistinguishable from controls, appropriately adjusting instrumental performance after outcome devaluation.

Glutamate mGluR2/3 agonists, which restore feedback on presynaptic release, also block expression of sensitization or reinstatement of cocaine seeking (Kim and Vezina, 2002; Peters and Kalivas, 2006) and interact with NAC effects (Moran et al, 2005). The observed cocaine-induced increase in EPSC frequency but not in amplitude is consistent with presynaptic changes in glutamate transmission caused by cocaine exposure. NAC may normalize presynaptic modulation of glutamate release by increasing basal glutamate, thereby restoring tone to mGluR $2 / 3$ receptors to inhibit 

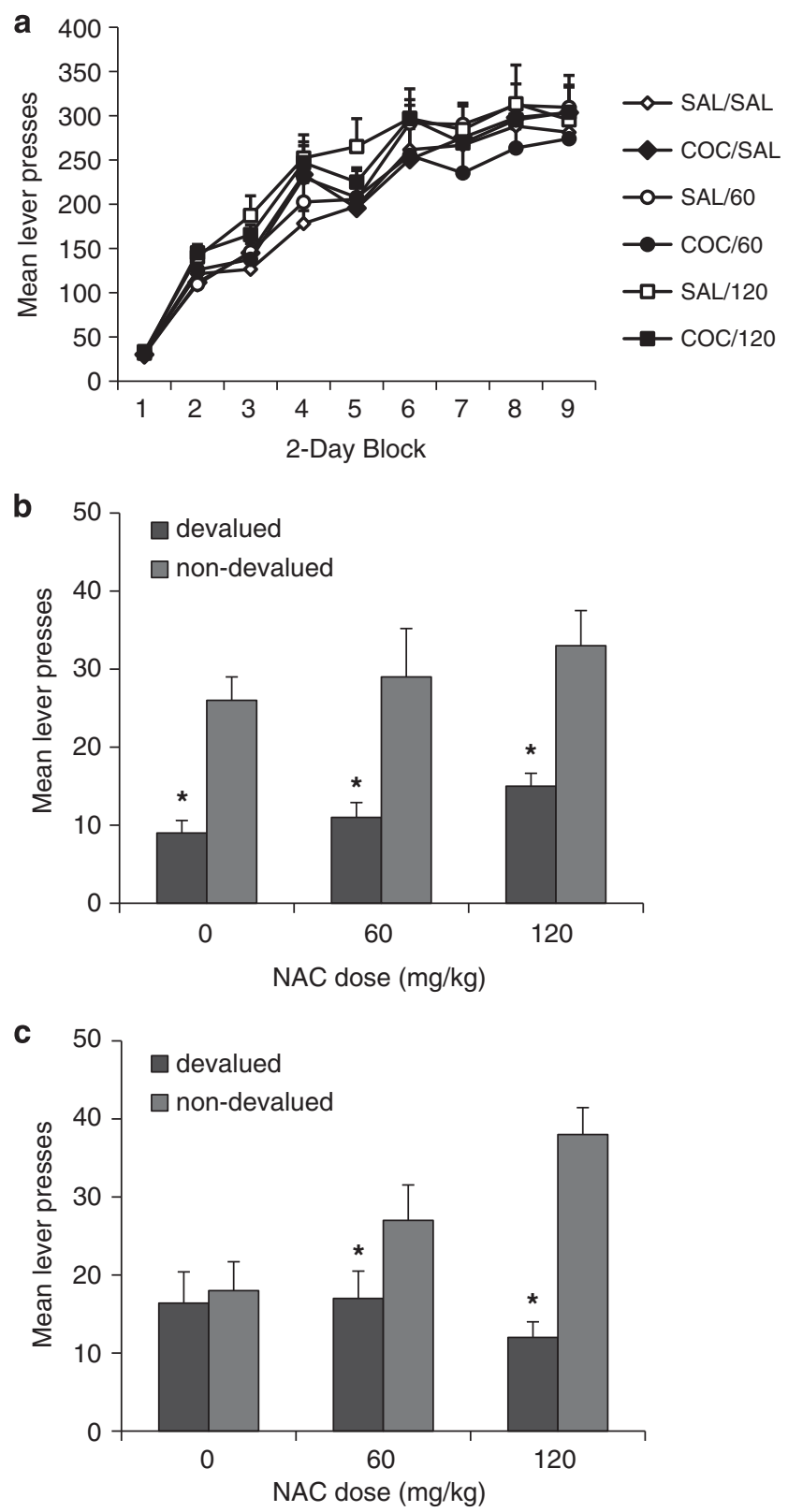

Figure 4 Effects of cocaine on sensitivity to outcome devaluation and interaction with NAC treatment. (a) Mean (+SEM) lever presses across 2day blocks of instrumental training. There were no group differences indicating equivalent performance in training. (b) Mean (+SEM) lever presses following outcome devaluation in groups treated with saline and 0 , 60 , or $120 \mathrm{mg} / \mathrm{kg}$ of NAC. All groups showed similar sensitivity to outcome devaluation, demonstrating that NAC alone had no detectable effect on devaluation. (c) Mean (+ SEM) lever presses following outcome devaluation in groups treated with cocaine $(30 \mathrm{mg} / \mathrm{kg})$ along with 0,60 , or $120 \mathrm{mg} /$ $\mathrm{kg}$ NAC. Animals receiving cocaine and $0 \mathrm{mg} / \mathrm{kg}$ were not sensitive to outcome devaluation, indicating that their performance was under habitual control. This effect was reversed when animals were cotreated with NAC (60 or $120 \mathrm{mg} / \mathrm{kg}$ ). $N=10-1 \mid$ rats per group, *significant devaluation effect.

synaptic release. Thus, it is likely that mGluR $2 / 3$ agonists would similarly prevent the accelerated habit formation observed following psychostimulant exposure.

Although an effect of NAC on cystine-glutamate exchange within the DMS is likely, NAC also upregulates GLT-1 expression (Knackstedt et al, 2010), an adaptation that may also serve to dampen glutamate release and could also normalize DMS activity. Future experiments will be needed to directly assess changes in cystine-glutamate exchange or GLT-1 function within the dorsal striatum following cocaine exposure, and it is likely that drugs that target GLT-1 function (eg, ceftriaxone; Trantham-Davidson et al, 2012) would also promote goal-directed control. Furthermore, NAC has numerous other physiological effects including effects on oxidative stress, mitochondrial function, inflammation, neurotrophins, and apoptosis, all of which may be relevant to psychiatric disease (Samuni et al, 2013). In relation to the current experiments, cocaine exposure produces strong glutamate and dopamine release, both of which in excess increase oxidative stress, altering neuronal function and in the extreme case lead to neuronal damage, effects that could lead to cognitive impairment (Berk et al, 2013). NAC ameliorates such effects by regulating transporter levels, modulating intracellular calcium, and reducing reactive oxygen species (Berk et al, 2013). In addition to providing cystine to drive cystine-glutamate exchange and directly affect glutamate signaling as described above, NAC increases cysteine levels that increases synthesis of glutathione, an important endogenous antioxidant that not only scavenges reactive oxygen species but also modulates NMDA receptor function and indirectly affects glutamate signaling. Thus, it is possible that some of the observed effects of NAC relate to these functions rather than, or in addition to, the proposed effects on glutamate homeostasis, yet the observed ability of NAC to normalize presynaptic glutamate release within the DMS, the region critical for goal-directed learning, suggests that regulation of glutamate signaling is likely to contribute to the effects of NAC on habit learning following cocaine.

\section{Functional Implications}

Over the course of drug use, many of the actions involved in acquiring and taking drugs become automatic, triggered by drug-related stimuli, difficult to control, and regulated largely outside conscious awareness (Tiffany, 1999). A developing automaticity of responding may help explain the transition from casual to frequent drug use and failures of behavioral control. Although habit-based performance itself does not equate with compulsive drug seeking, drug exposure appears to bring with it a rapid loss in sensitivity to the consequences of one's actions that may be an initial step in the progression to addiction (Pierce and Vanderschuren, 2010).

We examined the effects of experimenter-administered cocaine on subsequent learning, whereas several previous findings using self-administration paradigms have demonstrated that cocaine self-administration itself becomes habitual and has an increasing reliance of the DLS with extended training. Neural activation and dopamine efflux in the DLS increase over the course of self-administration training (Porrino et al, 2004; Ito et al, 2002). Furthermore, inactivation or dopamine antagonism specifically within the DLS reduce cocaine seeking after extended training and periods of abstinence (Vanderschuren et al, 2005; Fuchs et al, 2006), and disconnection achieved with lesions of the nucleus accumbens core and dopamine antagonism in the 
DLS reduce cocaine seeking after extended self-administration training, but is without effect on a distinct minimally trained response (Belin and Everitt, 2008). Thus, together, multiple sources of evidence suggest that DLS plays an increasing role in controlling drug-seeking behaviors. Nevertheless, although demonstrating increasing reliance on the DLS, few studies have explicitly examined whether cocaine self-administration is under habitual control. One study used self-administration of oral cocaine and found that a response earning a cocaine-sucrose solution was less sensitive to devaluation than a response earning lemonsucrose (Miles et al, 2003). Intravenous self-administration paradigms are, however, far more commonly used and the standard devaluation procedures used with natural rewards are not easily applied. Although intravenous cocaine selfadministration can be reduced by satiety (Norman and Tsibulsky, 2006), the effects of a cocaine 'satiety' treatment are difficult to interpret in terms of goal-directed $v s$ habitual performance because of the psychomotor effects of cocaine and the fact that self-administration rates relate to drug levels (Pickens and Thompson, 1968). Zapata et al (2010) avoided these issues by examining the effects of 'devaluation' achieved by extinguishing a drug-taking response in a seeking-taking chain (see also Olmstead et al, 2001). They found that performance of the seeking response was reduced following extinction of the taking response after limited but not extended training, thus providing direct evidence that cocaine self-administration becomes habitual over the course of extended training. Furthermore, they found that lidocaine infusions into the DLS restored sensitivity to devaluation following extended training consistent with previous demonstrations with natural reward (eg, Yin et al, 2004). Although the effects of DLS inactivation early in training were not assessed, other works examining the role of dorsal striatal subregions across the course of alcohol self-administration demonstrate that the DLS supports responding after extended but not moderate training (Corbit et al, 2012). In summary, multiple sources of evidence suggest that drug self-administration becomes habitual over extended training and has an increasing reliance on the DLS and associated circuitry; thus, the accelerated habit learning following psychostimulant exposure demonstrated by ourselves and others (Nelson and Killcross, 2006; Nordquist et al, 2007; LeBlanc et al, 2013) is not likely to be limited to experimenter-administered drug. Nevertheless, an explicit comparison of the rate of habit learning induced by self-administered drug relative to an appropriate control has yet to be conducted.

Although drug self-administration procedures may have greater face validity as a model of loss of control over drug use, it is not just decisions about drug seeking but a general rigidity in behavioral control that we believe is at the heart of the addictive process. Whereas much previous research has modeled drug self-administration and relapse to drug seeking, the current study demonstrates that exposure to cocaine accelerates habit learning and these effects are not limited to drug use itself. We also provide novel evidence that drug-induced habits can be prevented by NAC. The increased tendency for behavior, drug related or not, to fall under habitual control may present a significant obstacle for generating behavioral change. In addition to the established ability of NAC to reduce cocaine craving and relapse risk, its potential effects on goal-directed decision making in drug users may improve the efficacy of other types of intervention, such as cognitive behavioral therapy, and together produce better treatment outcomes.

\section{FUNDING AND DISCLOSURE}

The authors declare no conflict of interest.

\section{ACKNOWLEDGEMENTS}

The research reported in this paper was supported by an establishment grant from the University of Sydney to LHC and a Laureate Fellowship from the Australian Research Council to BWB.

\section{REFERENCES}

Adams CD (1982). Variations in the sensitivity of instrumental responding to reinforcer devaluation. Q J Exp Psychol 34B: 77-98.

Amen SL, Piacentine LB, Ahmad ME, Li SJ, Mantsch JR, Risinger $\mathrm{RC}$ et al (2011). Repeated $\mathrm{N}$-acetyl cysteine reduces cocaine seeking in rodents and craving in cocaine-dependent humans. Neuropsychopharmacology 36: 871-878.

Baker DA, McFarland K, Lake RW, Shen H, Tang XC, Toda S et al (2003). Neuroadaptations in cystine-glutamate exchange underlie cocaine relapse. Nat Neurosci 6: 743-749.

Balleine BW, O'Doherty JP (2010). Human and rodent homologies in action control: corticostriatal determinants of goal-directed and habitual action. Neuropsychopharmacology 35: 48-69.

Belin D, Everitt BJ (2008). Cocaine seeking habits depend upon dopamine-dependent serial connectivity linking the ventral with the dorsal striatum. Neuron 57: 432-441.

Berk M, Malhi GS, Gray LJ, Dean OM (2013). The promise of $\mathrm{N}$-acetylcysteine in neuropsychiatry. Trends Pharmacol Sci 34: 167-177.

Corbit LH, Balleine BW (2011). The general and outcome-specific forms of Pavlovian-instrumental transfer are differentially mediated by the nucleus accumbens core and shell. J Neurosci 31: 11786-11794.

Corbit LH, Janak PH (2010). Posterior dorsomedial striatum is critical for both selective instrumental and Pavlovian reward learning. Eur J Neurosci 31: 1312-1321.

Corbit LH, Muir JL, Balleine BW (2001). The role of the nucleus accumbens in instrumental conditioning: evidence of a functional dissociation between accumbens core and shell. J Neurosci 21: 3251-3260.

Corbit LH, Nie H, Janak PH (2012). Habitual alcohol seeking: time course and the contribution of subregions of the dorsal striatum. Biol Psychiatry 72: 389-395.

Fuchs RA, Branham RK, See RE (2006). Different neural substrates mediate cocaine seeking after abstinence versus extinction training: a critical role for the dorsolateral caudate-putamen. J Neurosci 26: 3584-3588.

Ghasemzadeh MB, Permenter LK, Lake R, Worley PF, Kalivas PW (2003). Homer1 proteins and AMPA receptors modulate cocaine-induced behavioural plasticity. Eur J Neurosci 18: 1645-1651.

Ito R, Dalley JW, Robbins TW, Everitt BJ (2002). Dopamine release in the dorsal striatum during cocaine-seeking behavior under the control of a drug-associated cue. J Neurosci 22: 6247-6253.

Kalivas PW (2009). The glutamate homeostasis hypothesis of addiction. Nat Rev Neurosci 10: 561-572. 
Kauer JA, Malenka RC (2007). Synaptic plasticity and addiction. Nat Rev Neurosci 8: 844-858.

Kim JH, Vezina P (2002). The mGlu2/3 receptor agonist LY379268 blocks the expression of locomotor sensitization by amphetamine. Pharmacol Biochem Behav 73: 333-337.

Knackstedt LA, Melendez RI, Kalivas PW (2010). Ceftriaxone restores glutamate homeostasis and prevents relapse to cocaine seeking. Biol Psychiatry 67: 81-84.

Kupchik YM, Moussawi K, Tang XC, Wang X, Kalivas BC, Kolokithas $\mathrm{R}$ et al (2012). The effect of $\mathrm{N}$-acetylcysteine in the nucleus accumbens on neurotransmission and relapse to cocaine. Biol Psychiatry 71: 978-986.

Laurent V, Leung B, Maidment N, Balleine BW (2012). mu- and delta-opioid-related processes in the accumbens core and shell differentially mediate the influence of reward-guided and stimulus-guided decisions on choice. J Neurosci 32: 1875-1883.

LeBlanc KH, Maidment NT, Ostlund SB (2013). Repeated cocaine exposure facilitates the expression of incentive motivation and induces habitual control in rats. PLoS One 8: e61355.

Li Y, Vartanian AJ, White FJ, Xue CJ, Wolf ME (1997). Effects of the AMPA receptor antagonist NBQX on the development and expression of behavioral sensitization to cocaine and amphetamine. Psychopharmacology (Berl) 134: 266-276.

Madayag A, Lobner D, Kau KS, Mantsch JR, Abdulhameed O, Hearing $\mathrm{M}$ et al (2007). Repeated $\mathrm{N}$-acetylcysteine administration alters plasticity-dependent effects of cocaine. J Neurosci 27: 13968-13976.

Miles FJ, Everitt BJ, Dickinson A (2003). Oral cocaine seeking by rats: action or habit? Behav Neurosci 117: 927-938.

Moran MM, McFarland K, Melendez RI, Kalivas PW, Seamans JK (2005). Cystine/glutamate exchange regulates metabotropic glutamate receptor presynaptic inhibition of excitatory transmission and vulnerability to cocaine seeking. J Neurosci 25: 6389-6393.

Moussawi K, Pacchioni A, Moran M, Olive MF, Gass JT, Lavin A et al (2009). N-Acetylcysteine reverses cocaine-induced metaplasticity. Nat Neurosci 12: 182-189.

Moussawi K, Zhou W, Shen H, Reichel CM, See RE, Carr DB et al (2011). Reversing cocaine-induced synaptic potentiation provides enduring protection from relapse. Proc Natl Acad Sci USA 108: 385-390.

Nelson A, Killcross S (2006). Amphetamine exposure enhances habit formation. J Neurosci 26: 3805-3812.

Nelson AJ, Killcross S (2013). Accelerated habit formation following amphetamine exposure is reversed by D1, but enhanced by D2, receptor antagonists. Front Neurosci 7: 76.

Nordquist RE, Voorn P, de Mooij-van Malsen JG, Joosten RN, Pennartz CM, Vanderschuren LJ (2007). Augmented reinforcer value and accelerated habit formation after repeated amphetamine treatment. Eur Neuropsychopharmacol 17: 532-540.

Norman AB, Tsibulsky VL (2006). The compulsion zone: a pharmacological theory of acquired cocaine self-administration. Brain Res 1116: 143-152.

Olmstead MC, Lafond MV, Everitt BJ, Dickinson A (2001). Cocaine seeking by rats is a goal-directed action. Behav Neurosci 115: 394-402.

Paulson PE, Robinson TE (1995). Amphetamine-induced timedependent sensitization of dopamine neurotransmission in the dorsal and ventral striatum: a microdialysis study in behaving rats. Synapse 19: 56-65.

Peters J, Kalivas PW (2006). The group II metabotropic glutamate receptor agonist, LY379268, inhibits both cocaine- and food-seeking behavior in rats. Psychopharmacology (Berl) 186: 143-149.

Pickens R, Thompson T (1968). Cocaine-reinforced behavior in rats: effects of reinforcement magnitude and fixed-ratio size. J Pharmacol Exp Ther 161: 122-129.

Pierce RC, Kalivas PW (1997). A circuitry model of the expression of behavioral sensitization to amphetamine-like psychostimulants. Brain Res Brain Res Rev 25: 192-216.

Pierce RC, Vanderschuren LJ (2010). Kicking the habit: the neural basis of ingrained behaviors in cocaine addiction. Neurosci Biobehav Rev 35: 212-219.

Porrino LJ, Lyons D, Smith HR, Daunais JB, Nader MA (2004). Cocaine self-administration produces a progressive involvement of limbic, association, and sensorimotor striatal domains. J Neurosci 24: 3554-3562.

Samuni Y, Goldstein S, Dean OM, Berk M (2013). The chemistry and biological activities of $\mathrm{N}$-acetylcysteine. Biochim Biophys Acta 1830: 4117-4129.

Schoenbaum G, Setlow B (2005). Cocaine makes actions insensitive to outcomes but not extinction: implications for altered orbitofrontal-amygdalar function. Cereb Cortex 15: 1162-1169.

Stewart J, Druhan JP (1993). Development of both conditioning and sensitization of the behavioral activating effects of amphetamine is blocked by the non-competitive NMDA receptor antagonist, MK-801. Psychopharmacology (Berl) 110: 125-132.

Tiffany ST (1999). Cognitive concepts of craving. Alcohol Res Health 23: 215-224.

Trantham-Davidson H, LaLumiere RT, Reissner KJ, Kalivas PW, Knackstedt LA (2012). Ceftriaxone normalizes nucleus accumbens synaptic transmission, glutamate transport, and export following cocaine self-administration and extinction training. J Neurosci 32: 12406-12410.

Vanderschuren LJ, Di Ciano P, Everitt BJ (2005). Involvement of the dorsal striatum in cue-controlled cocaine seeking. J Neurosci 25: 8665-8670.

Vanderschuren LJ, Kalivas PW (2000). Alterations in dopaminergic and glutamatergic transmission in the induction and expression of behavioral sensitization: a critical review of preclinical studies. Psychopharmacology (Berl) 151: 99-120.

Yin HH, Knowlton BJ, Balleine BW (2004). Lesions of dorsolateral striatum preserve outcome expectancy but disrupt habit formation in instrumental learning. Eur J Neurosci 19: 181-189.

Yin HH, Knowlton BJ, Balleine BW (2005a). Blockade of NMDA receptors in the dorsomedial striatum prevents action-outcome learning in instrumental conditioning. Eur J Neurosci 22: 505-512.

Yin HH, Ostlund SB, Knowlton BJ, Balleine BW (2005b). The role of the dorsomedial striatum in instrumental conditioning. Eur J Neurosci 22: 513-523.

Zapata A, Minney VL, Shippenberg TS (2010). Shift from goaldirected to habitual cocaine seeking after prolonged experience in rats. J Neurosci 30: 15457-15463.

Zhou W, Kalivas PW (2008). N-acetylcysteine reduces extinction responding and induces enduring reductions in cue- and heroininduced drug-seeking. Biol Psychiatry 63: 338-340. 